\title{
NEMA NU2-2001 Guided Performance Evaluation of Four Siemens ECAT PET Scanners
}

\author{
Hans Herzog, Lutz Tellmann, Carsten Hocke, Uwe Pietrzyk, Member, IEEE, Michael E. Casey, Member, IEEE, \\ and Torsten Kuwert
}

\begin{abstract}
The NEMA NU 2-2001 protocol has been especially designed for the performance evaluation of whole-body positron emission tomography (PET) measurements with scanners acquiring data in three-dimensional (3-D) mode without septa or with septa retracted. We report on the comparative NEMA NU 2-2001 performance tests on four different Siemens/CTI ECAT PET scanners, the two bismuth germanate oxide scanners HR+ and EXACT and the two lutetium oxyorthosilicate scanners ACCEL and EMERGE. All scanners were operated in 3-D mode only. Until now, no complete NEMA NU 2-2001 evaluations have been reported for the HR+ and EXACT. For the ACCEL and the EMERGE, these are the first published performance characteristics as far as we know. The HR+ showed a superior spatial resolution, whereas the ACCEL had the best countrate [noise equivalent count (NEC)] performance above a radioactivity concentration of $2 \mathrm{kBq} / \mathrm{cc}$. The sensitivity of the full-ring scanners did not differ much with numbers between 5982 and $7180 \mathrm{cps} / \mathrm{MBq}$. The sensitivity of the partial-ring scanner EMERGE was 2279 cps/MBq. Although the EMERGE yields lower NEC rates than the EXACT for radioactivity concentrations less than $10 \mathrm{kBq} / \mathrm{cc}$, it is favorable beyond this cross over point. The scatter fraction of all four scanners was about $48 \%$. The lesions to be examined by the image quality test could be seen best with the ACCEL. The NEMA NU 2-2001 performance tests proved to be a valuable guideline for this instrumental comparison.
\end{abstract}

Index Terms-NEMA-NU2 2001, performance test, positron emission tomography (PET).

\section{INTRODUCTION}

$\mathbf{T}$ HE INTRODUCTION of a new positron emission tomography (PET) scanner is usually accompanied by a paper that describes the technical specification and reports on the characteristic performance of the new instrument. In order to allow a comparable evaluation of different devices, measurement protocols have been developed and recommended by committees in which experienced research groups and industrial developers are represented. With regard to PET instrumentation, recommendations for standardized performance tests have been published by a task-group of the Society of Nuclear Medicine [1], the National Electrical Manufacturers Association (NEMA), and the International Electrotechnical Commission (IEC), which got input from a European Economic

Manuscript received November 15, 2003; revised June 2, 2004.

H. Herzog, L. Tellmann, and U. Pietrzyk are with the Institute of Medicine, Forschungszentrum Juelich, D-52425 Juelich, Germany (e-mail: h.herzog@ fz-juelich.de).

C. Hocke and T. Kuwert are with the Clinic of Nuclear Medicine, University of Erlangen, D-91054 Erlangen, Germany.

M. E. Casey is with CPS Innovations, Knoxville, TN 37932 USA.

Digital Object Identifier 10.1109/TNS.2004.835778
Community (EEC)-concerted action [2]. The former NEMA standard [3] and the IEC standard [4] suggested protocols for scanners of the third and fourth PET scanner generation [5], which were primarily used in two-dimensional acquisition mode, but optionally also in three-dimensional (3-D) mode without septa. With the increasing fraction of whole-body scans for PET oncology done in 3-D mode during the last years, those protocols proved to be rather limited in examining and comparing scanner performance with regard to the 3-D acquisition. The 3-D mode has not only the advantage of an increased sensitivity, but is also prone to a considerable increase in random and scattered events. Especially random coincidences increase dramatically in whole-body scanning, where they originate from body parts outside the scanner's field of view. As both the NU 2-1994 and IEC protocols did not adequately meet the radioactivity distribution, which is relevant for whole-body scanning, the NEMA-committee has developed a new protocol [6], which specifically takes into account the conditions of whole-body scanning.

The NU 2-2001 protocol includes a number of tests to check the different aspects of scanner performance. These aspects comprise spatial resolution (test i), scatter fraction, count losses, and randoms measurements (including the calculation of noise equivalent count (NEC) rates) (test ii), sensitivity (test iii), accuracy of corrections for count losses and randoms (test iv), and image quality, accuracy of attenuation, and scatter corrections (test v). To perform the necessary measurements, appropriate procedures and phantoms, which had already been suggested for testing the 3-D PET performance, were taken over, e.g., the sensitivity test developed by Bailey et al. [7]. In addition, a new cylindrical phantom of $70 \mathrm{~cm}$ length was designed, which is intended to simulate the distribution of random and scatter radiation, found in whole-body scans, more adequately than the former tests did.

In this paper, we followed the NU 2-2001 protocol to examine four different scanners of one manufacturer, i.e., Siemens/CTI. Two of them are widely distributed scanners based on bismuth germanate oxide (BGO) detectors, the ECAT EXACT and the ECAT HR+. The other two are the more recent scanners ECAT ACCEL and ECAT EMERGE which use lutetium oxyorthosilicate (LSO) crystals. The first of the latter two resembles the ECAT EXACT, but is equipped with LSO-rather than BGO-crystals, whereas the ECAT EMERGE is the LSO version of the partial ring PET scanner ECAT ART. Both BGO scanners have been evaluated using the older test standards [8]-[10]. Part of the NU 2-2001 protocol has already been applied to the EXACT and HR+ [11], [12]. 
TABLE I

SURVEY OF SCANNER SPECIFICATIONS

\begin{tabular}{l|c|c|c|c}
\hline & \multicolumn{4}{|c}{ Scanner } \\
\hline Parameter & HR+ & EXACT & ACCEL & EMERGE \\
\hline \hline Detector Material & BGO & BGO & LSO & LSO \\
\hline $\begin{array}{l}\text { Crystal Dimensions } \\
\left(\mathrm{mm}^{3}\right)\end{array}$ & $4.05 \times 4.39 \times 30$ & $6.75 \times 6.75 \times 20$ & $6.45 \times 6.45 \times 25$ & $6.45 \times 6.45 \times 25$ \\
\hline $\begin{array}{l}\text { Detector Ring } \\
\text { Diameter (cm) }\end{array}$ & 82.4 & 82.4 & 82.4 & 82.4 \\
\hline $\begin{array}{l}\text { Transverse } \\
\text { Field of View (cm) }\end{array}$ & 58.3 & 58.3 & 58.3 & 58.3 \\
\hline $\begin{array}{l}\text { Axial Field of View } \\
\text { (mm) }\end{array}$ & 155 & 162 & 162 & 162 \\
\hline $\begin{array}{l}\text { Image Planes } \\
\text { Plane Spacing (mm) }\end{array}$ & 63 & 47 & 47 & 47 \\
\hline $\begin{array}{l}\text { Coincidence Time } \\
\text { Window (nsec) }\end{array}$ & 12 & 3.375 & 3.375 & 3.375 \\
\hline
\end{tabular}

For the ACCEL and the EMERGE, this paper presents the first published performance characteristics as far as we know. We had the opportunity to perform all NU 2-2001 tests on the four scanners. Here, we report on our measurements and results. Because the EMERGE can acquire data in the 3-D mode only and because the 3-D mode is increasingly used in whole-body scanning, only 3-D data were recorded and are presented here.

\section{Material AND MethodS}

The recipes of the different tests are detailed in the "NEMA Standard Publication NU 2-2001: Performance Measurements of Positron Emission Tomographs" [6]. Furthermore, they have been explained by Daube-Witherspoon et al. [12] whose paper also described some additional comparative tests including the GE Advance and HR+ scanners. Here, we do not repeat the description of the performance tests, but report only on those details that differ from the standard approach and/or are specific for the scanners we have investigated.

\section{A. Scanner Description}

The ECAT HR+ has been located at the Institute of Medicine of the Forschungszentrum Juelich, Germany, since 1996. The EMERGE was installed at the Clinic of Nuclear Medicine, University of Erlangen, Germany, in September 2002. The EXACT has been in use at the St. Franziskus Hospital, Moenchengladbach, Germany, since January 2002, and the ACCEL at the Department of Nuclear Medicine at the Academic Hospital of the Free University of Brussels, Belgium since July 2001. Most of the technical specifications of the scanners have been detailed elsewhere (see manufacturer's technical specifications and [8]-[10]). Table I summarizes the major parameters characterizing the individual scanners. All scanners were used with the scanner-specific standard settings of the energy discrimination window, coincidence window, ring differences, as well as angular (mash) and axial (span) smoothing. ECAT scanners provide an online random correction, which is based on the delayed window method [13]. In order to access the random counts separately, the data used for test ii were recorded in the "Prompts \& Delayeds" acquisition mode, which provide two sets of sinograms within the acquisition file: consecutive time frames contain sinograms with prompt and delayed (i.e., random) counts in an alternating way.

\section{B. Spatial Resolution}

Glass capillaries of $1 \mathrm{~mm}$ inner diameter in which $1 \mathrm{~mm}$ ${ }^{18} \mathrm{~F}$-solution was inserted were used as the point sources in this test. The sources were arranged in air as required. The sinograms were recorded over periods ranging from $60-86 \mathrm{~s}$, during which up to 180000 counts were collected. Point spread images were reconstructed without attenuation and scatter correction. The software used for this purpose was the one included in the ECAT 7.2 software package. As standard reconstruction procedures without any smoothing filters are suggested by the NEMA protocol, data were FORE-rebinned and reconstructed by filtered backprojection using the direct Fourier reconstruction [14] (therefore referred here as "DIFT" reconstruction) with a ramp filter (smallest available filter width). In order to achieve an adequate sampling of the point spread profile, which would have not been possible with the standard pixel size of $2 \mathrm{~mm}$, the point sources were reconstructed in brain mode with an image matrix of $256 \times 256$ pixels and a pixel size of $0.25 \mathrm{~mm} \times$ $0.25 \mathrm{~mm}$. For this purpose, a zoom factor of 10 was chosen. In brain mode, the sinogram is trimmed to half the diameter of the normal field of view (FOV). Because of this fine sampling, it was not regarded as necessary to perform the parabolic fit of the curve peak as suggested in the NU 2-2001 protocol. The results of this test were rather inconsistent. In some evaluations, the radial resolution measured at $\mathrm{r}=10 \mathrm{~cm}$ was smaller than the tangential, which should not be the case. Therefore, an alternative reconstruction procedure was tested and applied as well. This reconstruction method is the 3-D filtered backprojection (PROMIS algorithm [15]), which had been the standard reconstruction method of the ECAT 7.1 software package and is further-on referred as "3DFBP." It is still available as vector processor (VP)-based reconstruction. Using 3DFBP with a ramp filter and a cutoff $=0.5 /$ pixel, more consistent results were obtained.

\section{Scatter Fraction, Count Losses, and Randoms Measurements}

To adapt the measurements of "scatter fraction, count losses, and randoms coincidences" for whole-body imaging, the NEMA committee introduced a new cylindrical $70 \mathrm{~cm}$ long phantom. The NEMA protocol distinguishes between the total event rate (TOT), the random event rate (R), the scatter event (S), and the true event rate (T). TOT, which is the sum of T, R, and $\mathrm{S}$, is the same as the prompt event rate as called by CTI. Random events are also called delayeds by CTI, because in their scanners the delayed window subtraction method is applied to estimate and correct for the random events. Furthermore, CTI defines trues as prompts minus delayeds. If not stated otherwise, the word true rate is used here in the sense of the NEMA definition. For the purpose of the measurements reported here, a phantom according to the NEMA NU2-2001 instructions was produced by our workshop [16]. It consists of polyethelene with the required density of $0.96 \mathrm{~g} / \mathrm{cm}^{3}$, is $70 \mathrm{~cm}$ long, and has a diameter of $20 \mathrm{~cm}$. At a radius of $4.5 \mathrm{~cm}$, there is a hole with a diameter of $6.4 \mathrm{~mm}$ parallel to the phantom's axis. The 
tube in which the radioactivity is inserted is made of plexiglass with an outer diameter of $5 \mathrm{~mm}$ and an inner diameter of 3 $\mathrm{mm}$. The starting radioactivity concentration of ${ }^{18} \mathrm{~F}$ solution ranged from $24 \mathrm{kBq} / \mathrm{cc}(\mathrm{HR}+)$ to about $45 \mathrm{kBq} / \mathrm{cc}$ for the other scanners. The activities were measured with dose calibrators available at the individual sites that were routinely checked for accuracy. This radioactivity concentration is calculated as the radioactivity within the tube divided by the phantom volume of $22000 \mathrm{cc}$. Between 30 and 40 frames lasting $30 \mathrm{~min}$ each and separated by an interval of 25 min were recorded so that the total acquisition time corresponded to at least 15 halftimes of ${ }^{18} \mathrm{~F}$. The NU 2-2001 protocol recommends to continue the measurements until the random count rate is less than $1 \%$ of the true count rate, so that the scatter fraction can be derived from frames not influenced by randoms. This could not be realized in all four scanners. From high to low count rates, there was a steady decrease of the delayed/prompt ratio. At very low count rates, however, this ratio increased again, i.e., the random counts became nonnegligible. In the case of BGO scanners, the imperfectly shielded transmission line sources are responsible for the background due to random coincidences. In the case of LSO scanners, this background is caused by the natural abundance of ${ }^{176} \mathrm{Lu}$ in the LSO crystals. The so-called net-true coincidence counts (still comprising the scattered events) were obtained by subtracting the delayed (random) sinograms from the prompt sinograms. According to the NEMA protocol, all counts were evaluated only within a diameter of $24 \mathrm{~cm}$ and do not refer to the total field of view of the scanner. The scatter fraction was determined by exploiting those frames with the lowest delayed/prompt ratio. Frames at a radioactivity concentration of $0.5-1 \mathrm{kBq} / \mathrm{cc}$ were evaluated. Since all ECAT scanners use the delayed window subtraction method [13] to correct for random events, the NEC [17] rate was calculated according to the equation

$$
\mathrm{NEC}=\frac{T^{2}}{T+S+2 R}
$$

i.e., the so-called $2 \mathrm{R}-\mathrm{NEC}$ rates were obtained.

In order to add an interesting issue to the discussion, NEC rates of the two LSO scanners ACCEL and EMERGE were calculated for a hypothetical scatter fraction of $30 \%$. A value of $30 \%$ might be possible, if further instrumental progress will succeed to transfer the high light output of LSO into a smaller energy window, and consequently into a lower scatter fraction. To calculate the appropriate NEC data, the scatter fraction obtained by the measurement was replaced by the value of $30 \%$.

\section{Sensitivity}

Instead of five metal sleeves described in the NU 2-2001 protocol for the sensitivity test, we used only four, i.e., the thinnest sleeve was not used. For the four aluminum sleeves, which were produced by our workshop, slightly different diameters than suggested by the NU 2-2001 protocol had to be used [16]. The inner and outer diameters of the $70 \mathrm{~cm}$ long sleeves were 7 and $9.9 \mathrm{~mm}$ (sleeve 1), 10 and $11.9 \mathrm{~mm}$ (sleeve 2), 12 and 14.95 $\mathrm{mm}$ (sleeve 3), and 15 and $18 \mathrm{~mm}$ (sleeve 4), respectively. With these diameters, the sleeves fitted each other perfectly. The plastic tube containing the radioactivity had an inner diameter of $4 \mathrm{~mm}$ and an outer one of $6.5 \mathrm{~mm}$. The radioactivity inserted into this tube was approximately $5 \mathrm{MBq}^{18} \mathrm{~F}$. The activities were measured with dose calibrators and diluted as necessary. For each measurement, between 500000 and 800000 prompt counts were recorded with the "normal" acquisition mode, i.e., the sinograms were online random corrected by using the delayed window subtraction method [13]. As there are no scattered events in this test, the prompt events represent the true events. The individual measurements took from 20-100 $\mathrm{s}$, depending on the scanner's sensitivity and the radioactivity used. The 3-D sinograms were single-slice rebinned using a MATLAB program.

\section{E. Accuracy of Corrections for Count Losses and Randoms}

For this test, the measurements performed for "scatter fraction, count losses, and randoms measurements" were used. As stated above, a delayed/prompt ratio below $1 \%$ could not be achieved with the scanners examined here. Therefore, we extrapolated our data on the basis of the acquisition with the lowest achievable delayed/prompt ratio, which ranged from $6.3 \%$ to $10 \%$. Since the data were recorded in "Prompts \& Delayeds" acquisition mode which did not allow a direct image reconstruction of sinograms of (net-)true coincidence counts were obtained with a MATLAB program which subtracted delayed from prompt sinograms and created a random-corrected ECAT7 sinogram file. The sinograms were reconstructed with filtered backprojection (DIFT) using a Shepp filter with a width of $2.5 \mathrm{~mm}$, which is the standard reconstruction procedure applied to our non-whole-body studies. The reconstruction included attenuation, scatter, and decay correction. In all image planes, a ROI with a diameter of $18 \mathrm{~cm}$ was defined on the reconstructed transmission images with a concentric position over the phantom. These ROI were superimposed on the emission images, yielding an ROI average $\mathrm{R}_{\mathrm{ROI}, i, j}$ for each image plane $i$ and time frame $j$. The evaluation of this test had to be adapted to the standard outcome of reconstructed PET images produced by the ECAT7.2 software: the reconstruction program includes the calibration, so that the pixel content of the images is given in becquerels per cubic centimeter rather than in counts. Therefore, the numbers of radioactivity concentration were examined rather than the count rates, as suggested originally by the NEMA protocol. Furthermore, all images are decay-corrected in relation to the scan start time. Therefore, we calculated the relative count rate error of this test as follows:

$$
\Delta r_{i, j}=100\left(\frac{R_{\mathrm{ROI}, i, j}}{R_{\mathrm{REF}, i}}-1\right) \% .
$$

$\mathrm{R}_{\mathrm{Ref}, i}$ is the average of the radioactivity concentrations $\mathrm{R}_{\mathrm{ROI}, i, j}$ obtained for those three time frames with the lowest delayed/prompt ratio. For these frames, the dead time losses were approximately $1 \%$.

\section{F. Image Quality, Accuracy of Attenuation, and Scatter Corrections}

This test was done with the EEC whole-body phantom in which six spheres were installed. At the bottom of this phantom, the $70 \mathrm{~cm}$ cylinder phantom was placed whose tube was filled with approximately $90 \mathrm{MBq}^{18} \mathrm{~F}$ (only $35 \mathrm{MBq}$ for the ACCEL). 
TABLE II

RECONSTRUCTED IMAGE RESOLUTION (USING 3DFBP WITH A RAMP FILTER OF 0.5 CUTOFF)

\begin{tabular}{l|c|c|c|c|c|c|c|c}
\hline & \multicolumn{7}{|c|}{ Scanner } \\
\hline & \multicolumn{2}{|c|}{ HR+ } & \multicolumn{2}{c|}{ EXACT } & \multicolumn{2}{c|}{ ACCEL } & \multicolumn{2}{c}{ EMERGE } \\
\hline Radial Position (cm) & 1 & 10 & 1 & 10 & 1 & 10 & 1 & 10 \\
\hline Orientation & & & & & & & & \\
\hline Radial Resol. (mm) & 4.39 & 5.65 & 6.19 & 6.81 & 6.22 & 7.20 & 5.95 & 7.29 \\
\hline Tangential Resol. (mm) & 4.39 & 4.64 & 6.19 & 6.08 & 6.22 & 6.47 & 5.95 & 6.42 \\
\hline Axial Resol. (mm) & 5.10 & 5.33 & 5.16 & 6.16 & 5.71 & 6.70 & 6.51 & 7.68 \\
\hline
\end{tabular}

The NEMA protocol prescribes that the largest two spheres are filled with cold water, whereas the space outside the spheres (background) and the smaller four spheres are filled with radioactivity such that the ratio of radioactivity concentration of these spheres and the background is $4: 1$ in a first trial and $8: 1$ in a second trial. The radioactivity concentration of the background (BG) ranged from $2.8-7.5 \mathrm{kBq} / \mathrm{cc}$. The acquisition and the reconstruction procedure are to be performed similarly to those of a whole-body study. Therefore, the emission scan duration was 6 min in case of the HR+ and EXACT, 7 min in case of the ACCEL, and $8 \mathrm{~min}$ in case of the EMERGE. The transmission scan recorded with the phantom containing radioactivity took $4 \mathrm{~min}$ for the EMERGE and $3 \mathrm{~min}$ for the other scanners. The attenuation correction applied a segmented attenuation map. As common for the clinical studies, images were reconstructed with the attenuation-weighted ordered subset expectation maximization procedure [18] with eight subsets and two iterations followed by a Gaussian filtering with a filter width of $4 \mathrm{~mm}$. In order to check the accuracy of the attenuation and scatter corrections, the parameter $\Delta C_{\text {lung }}$ was determined. For this purpose, a cold rod with a density of $0.33 \mathrm{~g} / \mathrm{cm}^{3}$ was positioned at the phantom's center. This density was achieved by filling the rods with unboiled noodles [16]. According to the NEMA protocol, the residual error was calculated as the ROI average of the "lung" insert divided by the average of the BG ROIs.

\section{RESULTS}

\section{A. Spatial Resolution}

Table II summarizes the reconstructed image resolution expressed as full-width at half-maximum. The data shown were measured in air with a radial position of the point source at 1 and $10 \mathrm{~cm}$ from the center. Because of the inconsistent results obtained with the primary reconstruction method (DIFT) available in the ECAT 7.2 software, the results reported in Table II are derived from point spread images reconstructed with an older reconstruction software (3DFBP). As expected, the resolution of the HR+ was superior to those of the other scanners. Fig. 1 illustrates the different outcome of the ways in reconstructing the point spread images.

\section{B. Scatter Fraction, Count Losses, and Randoms Measurements}

The scatter fraction ranged from $46 \%$ (ACCEL) to $50 \%$ (EXACT), i.e., it was nearly the same for all the scanners (Table III). This can be expected, because the ring dimension

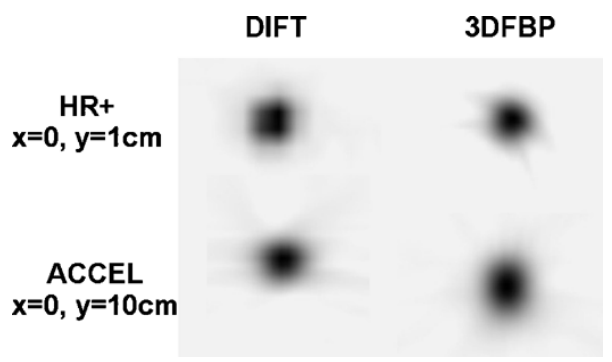

Fig. 1. Comparison of point spread images, which were used to determine the image resolution, reconstructed with either the present ECAT 7.2 software (DIFT) or the previous 3DFBP method. (Top) In contrast to the image obtained with DIFT, the 3DFBP reconstructed image has a circular shape, which is expected for the position $\mathrm{x}=0$ and $\mathrm{y}=1 \mathrm{~cm}$. (Bottom) In this case where the point source was located at $\mathrm{x}=0$ and $\mathrm{y}=10 \mathrm{~cm}$, only the image produced by the $3 \mathrm{DFBP}$ reconstruction showed the expected shape with the radial extension greater than the tangential one.

TABLE III

SCATTER FRACTION AND 2R-NEC RATES

\begin{tabular}{l|c|c|c|c}
\hline & \multicolumn{4}{|c}{ Scanner } \\
\hline Parameter & HR+ & EXACT & ACCEL & EMERGE \\
\hline Scatter Fraction (\%) & $46.9 \%$ & $49.8 \%$ & $46.3 \%$ & $48.8 \%$ \\
\hline $\begin{array}{l}\text { NEC Rate (cps) } \\
@ 2 \mathrm{kBq} / \mathrm{cc}\end{array}$ & 16800 & 12700 & 15100 & 5460 \\
\hline $\begin{array}{l}\text { Peak NEC Rate (cps) } \\
@ \text { Act.Conc. }(\mathrm{kBq} / \mathrm{cc})\end{array}$ & $26000 @ 8.3$ & $16400 @ 5.8$ & $46900 @ 18$ & $13300 @ 13$ \\
\hline
\end{tabular}

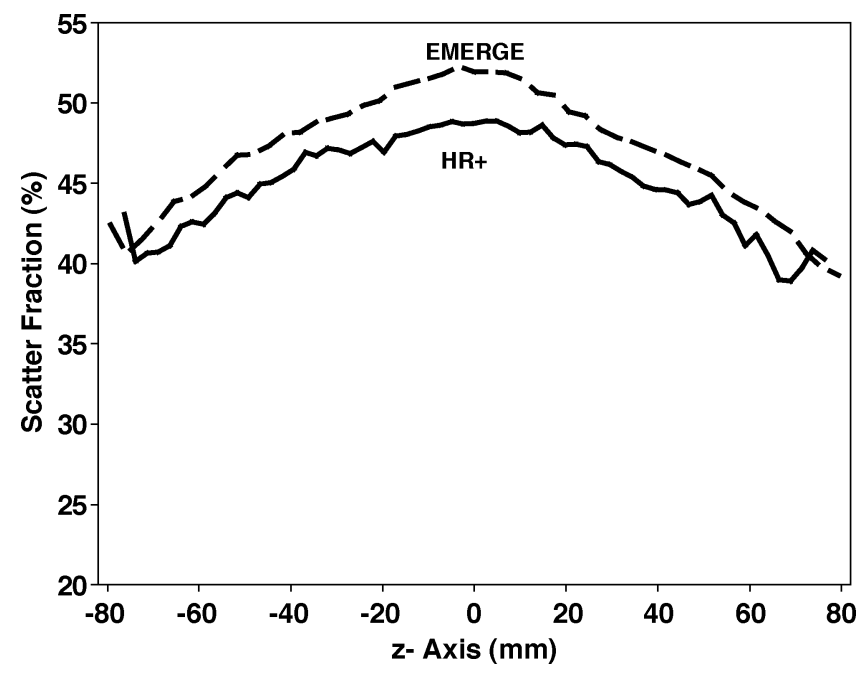

Fig. 2. Scatter fraction determined for the single planes is plotted as a function of the image planes for the ECAT scanners HR+ (63 planes) and EMERGE (47 planes). The positive $\mathrm{z}$ axis is directed to the scanner's front end.

of the scanners are nearly identical, and the energy window $(350-650 \mathrm{keV})$ was the same. Fig. 2 shows the scatter fraction along the $\mathrm{z}$ axis for the HR+ and the EMERGE scanner. The profiles of the EXACT and the ACCEL were similar to that of the EMERGE. As underlined by Fig. 2, the scatter fraction was maximum in the center of the field view. Compared to the minimum scatter fraction, which was found at or near the end of the field of view, the maximum scatter fraction was higher by a factor of 1.26 in case of the HR+ and 1.33 in case of the EMERGE. This number was about 1.3 for the other two scanners.

When looking at the low range of the total count rates (Fig. 3), the HR+, the ECAT, and the ACCEL are similar, which is also 


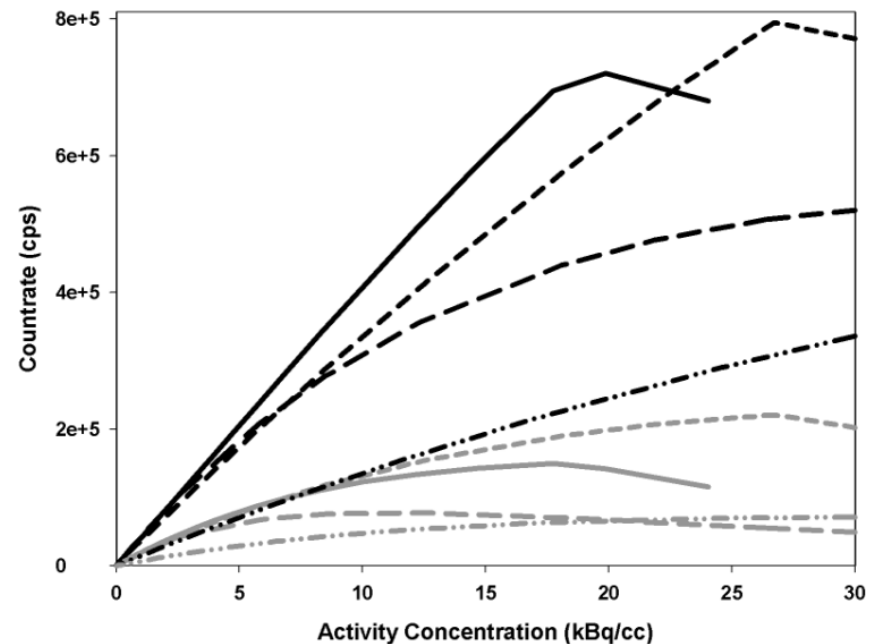

Fig. 3. Comparison of (black) the total and (dark gray) true count rates of the four ECAT scanners as a function of the average radioactivity concentration within the $70 \mathrm{~cm}$ long phantom. The counts were summed within a diameter of $24 \mathrm{~cm}$.

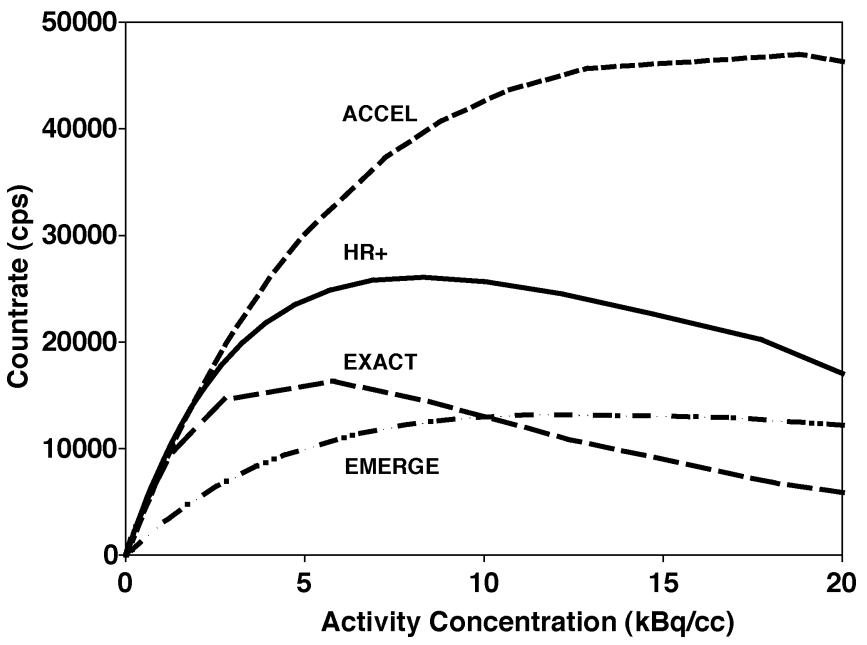

Fig. 4. Comparison of the 2R-NEC rates of the four ECAT scanners as a function of the average radioactivity concentration within the $70 \mathrm{~cm}$ long phantom.

mirrored by the sensitivity (see below), whereas the partial ring scanner EMERGE records a much lower count rate. The HR+ peaks with $720 \mathrm{kcps}$ at $20 \mathrm{kBq} / \mathrm{cc}$, the ACCEL with $795 \mathrm{kcps}$ at $27 \mathrm{kBq} / \mathrm{cc}$, the EXACT with $530 \mathrm{kcps}$ at $32 \mathrm{kBq} / \mathrm{cc}$. The much lower count rate of the EMERGE does not reach its limit at the radioactivity concentrations studied here.

As already explained above, the random count rate became not negligible at low radioactivity concentrations. The smallest possible delayed/prompt ratio was measured for the EXACT with $6.4 \%$. This number was $7.8 \%$ for the $\mathrm{HR}+, 10 \%$ for the ACCEL and $9.3 \%$ for the EMERGE. Below a radioactivity concentration of $2 \mathrm{kBq} / \mathrm{cc}$, the NEC rates of the HR+, EXACT, and ACCEL did not differ much. The NEC data of the individual scanners are shown in Fig. 4 and Table III, which also summarizes the peak NEC rates $\left(\mathrm{R}_{\mathrm{NEC} \text {,peak }}\right)$ with their corresponding radioactivity concentrations $a_{N E C, \text { peak }}$ at which $R_{N E C, p e a k}$ is reached. The LSO scanner ACCEL with its small coincidence window of $6 \mathrm{~ns}$ and consequently low random rate surpasses the $\mathrm{HR}+$ above a radioactivity concentration of $2 \mathrm{kBq} / \mathrm{cc}$. Its peak
TABLE IV

SENSITIVITY

\begin{tabular}{l|c|c|c|c}
\hline & \multicolumn{4}{|c}{ Scanner } \\
\hline & HR+ & EXACT & ACCEL & EMERGE \\
\hline $\begin{array}{l}\text { Sens. } \mathrm{r}=0 \mathrm{~cm} \\
(\mathrm{cps} / \mathrm{MBq})\end{array}$ & 6650 & 5982 & 6362 & 2279 \\
\hline $\begin{array}{l}\text { Sens. } \mathrm{r}=-10 \mathrm{~cm} \\
(\mathrm{cps} / \mathrm{MBq})\end{array}$ & 7180 & 6251 & 6329 & 2180 \\
\hline
\end{tabular}

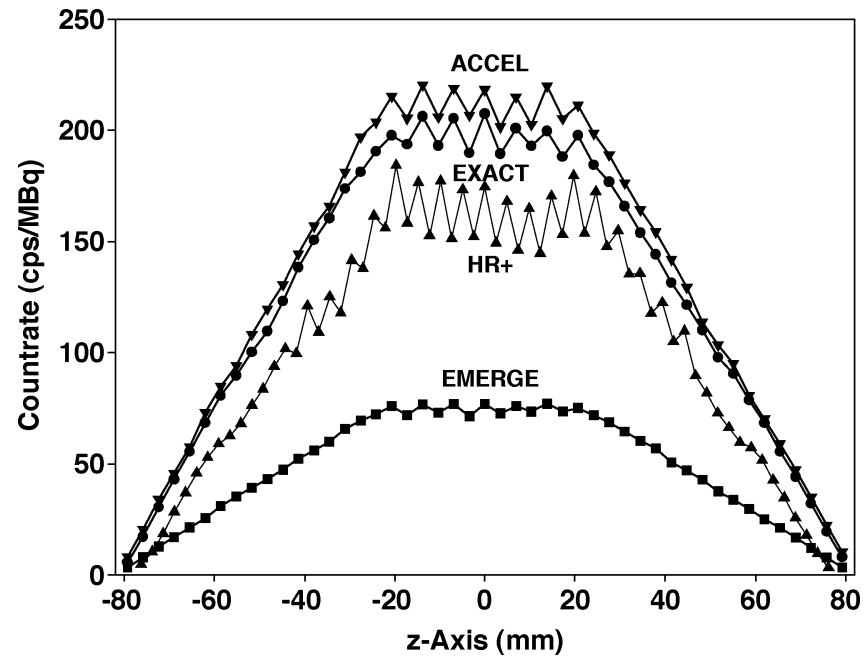

Fig. 5. Sensitivity of the single planes as a function along the scanner's $\mathrm{z}$ axis The countrate of a single plane was obtained after single slice rebinning of the original 3-D data. The positive $\mathrm{z}$ axis is directed to the scanner's front end.

NEC rate is nearly three times that of the EXACT and twice that of the $\mathrm{HR}+$. On the other hand, this peak rate is reached at rather high radioactivity concentrations. Both the full ring EXACT and the partial ring EMERGE show much lower NEC curves with a crossover point at $10 \mathrm{kBq} / \mathrm{cc}$. Above this concentration, the LSO-ART scanner becomes superior. To take advantage of this behavior, an increased dose of injected radioactivity has to be chosen for this scanner.

\section{Sensitivity}

Our tests showed that the 3-D sensitivity values of the EXACT, HR+, and the ACCEL do not differ much. The results are summarized in Table IV. The 3-D sensitivity of the HR+ is the highest with $6650 \mathrm{cps} / \mathrm{MBq}$. The EMERGE with its partial ring design shows $2279 \mathrm{cps} / \mathrm{MBq}$, which is $36 \%$ of the sensitivity of the ACCEL. For the two BGO scanners, the sensitivity was $5 \%$ higher when the sensitivity measurements were carried out with a $10 \mathrm{~cm}$ offset of the $\mathrm{Al}$ tubes, whereas it was unchanged or slightly decreased for the LSO scanners. Fig. 5 compares the sensitivity profiles of the scanners plotted against the $\mathrm{z}$ axis. The dead time losses were approximately $1 \%$. Due to the problem of random background, the random rate was between $5 \%$ and $10 \%$ of the difference prompt minus delayed ("CTI-true") event rate.

\section{Accuracy of Corrections for Count Losses and Randoms}

Fig. 6 shows the relative errors in radioactivity measured in the image plane in the middle of the FOV versus the radioactivity concentration within the $70 \mathrm{~cm}$ cylinder phantom. It illus- 


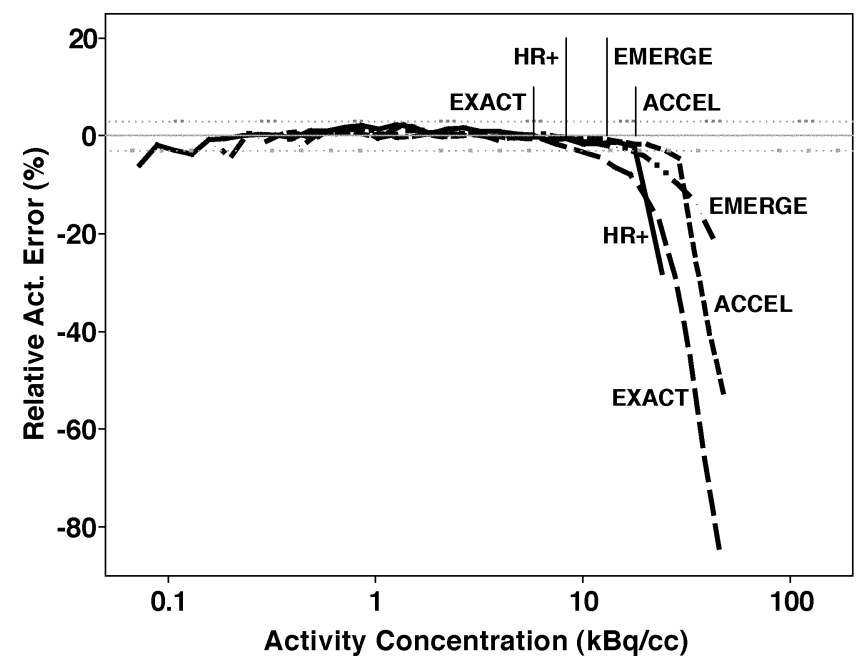

Fig. 6. Relative error in radioactivity measured from the image, which is determined according to (2), remains within a range of $+/-3 \%$ (indicated as horizontal gray dotted lines), if the recorded radioactivity concentration is below the radioactivity concentration $\mathrm{a}_{\mathrm{NEC} \text {, peak }}$, which is marked as vertical lines above the $+/-3 \%$ range.

trates that the errors remain within the $+/-3 \%$ range at radioactivity concentrations below that of the peak NEC rate (compare Table III). Only under the influence of bad count statistics at very low radioactivity the errors surpass this range slightly. When looking at all other planes and at radioactivity concentrations below aNEC,peak, the errors in case of the EXACT remain less than $6.3 \%$, of the HR+ less than $6.9 \%$, of the ACCEL less than $12.9 \%$, and of the EMERGE less than $8.5 \%$.

\section{E. Image Quality, Accuracy of Attenuation, and Scatter Corrections}

The main numbers resulting from the image quality test are summarized in Table V. The ACCEL yielded the lowest background variation, which is also demonstrated in Fig. 7, where it is compared with the other scanners in case of the $1: 8$ background to hot sphere ratio. On the other hand, the ACCEL had a comparably lower hot sphere contrast. Accordingly, Fig. 7 indicates that the detection of the three larger hot spheres is equally good in the other scanners. Because of the smooth background, the smallest $(10 \mathrm{~mm})$ sphere can be seen with the ACCEL, but not with the EXACT and EMERGE. This sphere was also visible on the HR+ image, which is concordant with the highest hot contrast of $12 \%$ found for the smallest sphere. However, this sphere might not have been detected without a knowledge of the phantom's structure.

When the $1: 4$ study was analyzed, only the two largest hot spheres (17 and $22 \mathrm{~mm}$ ) could be seen on the images of the $\mathrm{HR}+$, EXACT, and EMERGE, whereas the ACCEL revealed also the $13 \mathrm{~mm}$ sphere-again due to its smooth background. Although the ACCEL and the HR+ had the highest cold contrast, the cold spheres could be seen clearly only in the ACCEL images with their low background noise. This finding holds for both the " $1: 8$ " and " $1: 4$ " study. The values of $\Delta C_{\text {lung, which served }}$ to estimate the accuracy of the attenuation and scatter corrections, ranged from 29\% (EMERGE 1 : 8-test) to 48\% (ACCEL $1: 8$-test), demonstrating a considerable residual reconstructed radioactivity concentration in this cold area.

\section{DISCUSSION}

This paper presents a comparison of performance characteristics of four ECAT PET scanners manufactured by Siemens/CTI. The tests were performed according to the recommendations of the NEMA NU2-2001 protocol [6]. Originally, it was not intended to do a prospective examination of all four scanners. We started with first measurements on the $\mathrm{HR}+$ to get acquainted to the new standard. Afterward, it was agreed to do these measurements on a newly installed partial ring LSO scanner, the EMERGE. Only later we realized that it might be interesting to work on a more extended comparative examination and to include two further scanners, the older but widely used EXACT, and the recent full-ring LSO-PET ACCEL. Except for the newly installed EMERGE, no specific detector setup has been made prior to the tests. Thus, our test may not represent the optimum scanner's state that is expected at least at the time of delivery.

Most of the tests could be realized as suggested by the NEMA protocol. The main difference was the handling of those measurements (determination of the scatter fraction and sensitivity) for which negligible rates of randoms are demanded. For the reasons already mentioned above concerning scanner construction (transmission line sources) and detector material (including radioactive isotope ${ }^{176} \mathrm{Lu}$ ), it was not possible to fall below a delayed/prompt ratio of $5 \%$. On the other hand, the contribution of the delayed counts could be taken into account in test ii. Here the scatter fraction was derived from sinograms which had been recorded in the "Prompts \& Delayeds" acquisition mode so that the sinograms of the delayed coincidences could be subtracted from the prompt coincidences prior to the evaluation guided by Figure 4-2 of the NEMA protocol.

In this evaluation, the so-called $2 \mathrm{R}-\mathrm{NEC}$ rates were determined, since the random correction of the ECAT scanners uses the delayed window technique. In order to allow a better comparison with other scanners whose specifications refer to the $1 \mathrm{R}-\mathrm{NEC}$ rate, since their random correction is based on the use of single count rates or they apply a smoothing of the recorded delayed counts, Table VI lists the peak NEC rates for both the $2 \mathrm{R}$ - and 1R-case. The count rates of the HR+ and EXACT reported here are in agreement with the numbers reported elsewhere [11].

In the low count range, there are only small differences between the HR+, the EXACT, and the ACCEL, although the coincidence time window of the ACCEL is only 6 ns so that the delayed/prompt ratio of the ACCEL decreases to $17 \%$ at $5 \mathrm{kBq} / \mathrm{cc}$ compared to $32 \%$ for the EXACT. Thus, the improved NEC rate of the ACCEL with respect to its BGO sister EXACT is due to the lower random rate. It might be even higher with a lower scatter fraction, which should be expected because of the higher light output of LSO compared to BGO. The assumption of a $30 \%$ scatter fraction resulted in NEC rates shown in Fig. 8. This figure clearly indicates possible progress with the LSO scanners. With a scatter fraction of $30 \%$, the partial ring LSO scanner EMERGE would perform similarly as the full ring BGO scanner EXACT even in the low radioactivity range in spite of its inferior sensitivity.

It has been described above that the present standard reconstruction software is not suited to deliver proper results of res- 
TABLE V

RESUltS OF IMAGE QUALITY TEST

\begin{tabular}{l|c|c|c|c|c|c|c|c}
\hline & \multicolumn{9}{|c|}{ Scanner } \\
\hline Parameter & \multicolumn{2}{|c|}{ HR+ } & \multicolumn{2}{c|}{ EXACT } & \multicolumn{2}{c}{ ACCEL } & \multicolumn{2}{c}{ EMERGE } \\
\hline Background/Hot Sphere & $1: 8$ & $1: 4$ & $1: 8$ & $1: 4$ & $1: 8$ & $1: 4$ & $1: 8$ & $1: 4$ \\
\hline COV:Background-SD-37mm & $5.3 \%$ & $6.2 \%$ & $5.0 \%$ & $5.4 \%$ & $3.8 \%$ & $4.1 \%$ & $5.5 \%$ & $6.2 \%$ \\
\hline COV:Background-SD-10mm & $10.6 \%$ & $13.4 \%$ & $9.2 \%$ & $9.8 \%$ & $5.1 \%$ & $4.9 \%$ & $13.7 \%$ & $11.6 \%$ \\
\hline Hot Contrast $(22 \mathrm{~mm})$ & $43.1 \%$ & $46.2 \%$ & $34.2 \%$ & $64.8 \%$ & $35.9 \%$ & $37.2 \%$ & $49.9 \%$ & $60.3 \%$ \\
\hline Hot Contrast $(10 \mathrm{~mm})$ & $12.6 \%$ & $1.4 \%$ & $6.0 \%$ & $13.7 \%$ & $7.2 \%$ & $1.1 \%$ & $4.9 \%$ & $20.8 \%$ \\
\hline Cold Contrast $(37 \mathrm{~mm})$ & $56.4 \%$ & $54.1 \%$ & $40.2 \%$ & $44.8 \%$ & $55.5 \%$ & $57.0 \%$ & $46.9 \%$ & $44.8 \%$ \\
\hline Average $\Delta \mathrm{Clung}_{\text {lung }}$ & $34.3 \%$ & $34.0 \%$ & $40.7 \%$ & $42.3 \%$ & $47.8 \%$ & $44.3 \%$ & $28.7 \%$ & $29.9 \%$ \\
\hline
\end{tabular}
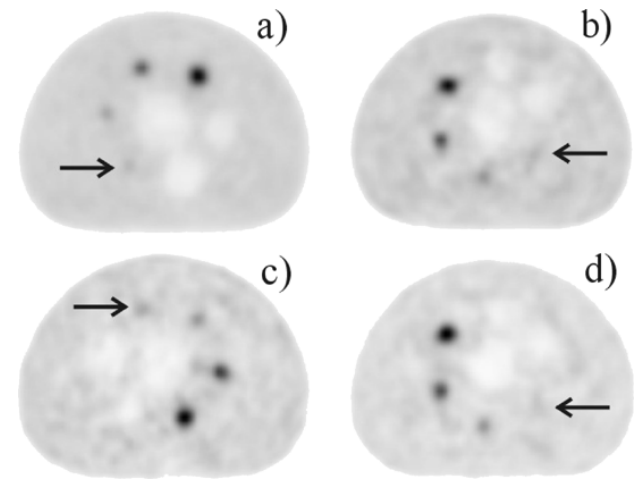

Fig. 7. Transverse images of the image quality test done with the background/hot sphere ratio of $1: 8$. The images show the section across the center of the spheres. (a) ACCEL. (b) EXACT. (c) HR+. (d) EMERGE. The $10 \mathrm{~mm}$ sphere is indicated by arrows.

TABLE VI

2R-NEC VERSUS 1R-NEC PEAK RATE

\begin{tabular}{l|c|c|c|c}
\hline & \multicolumn{4}{|c}{ Scanner } \\
\hline Parameter & HR+ & EXACT & ACCEL & EMERGE \\
\hline $\begin{array}{l}\text { Peak 2R-NEC-Rate } \\
(\mathrm{cps}) @ \text { Act.Conc. } \\
(\mathrm{kBq} / \mathrm{cc})\end{array}$ & $26000 @ 8.3$ & $16400 @ 5.8$ & $46900 @ 18$ & $13300 @ 13$ \\
\hline $\begin{array}{l}\text { Peak 1R-NEC-Rate } \\
(\mathrm{cps}) @ \text { Act.Conc. } \\
(\mathrm{kBg} / \mathrm{cc})\end{array}$ & $37000 @ 10$ & $22200 @ 6.3$ & $63000 @ 22$ & $19000 @ 17$ \\
\hline
\end{tabular}

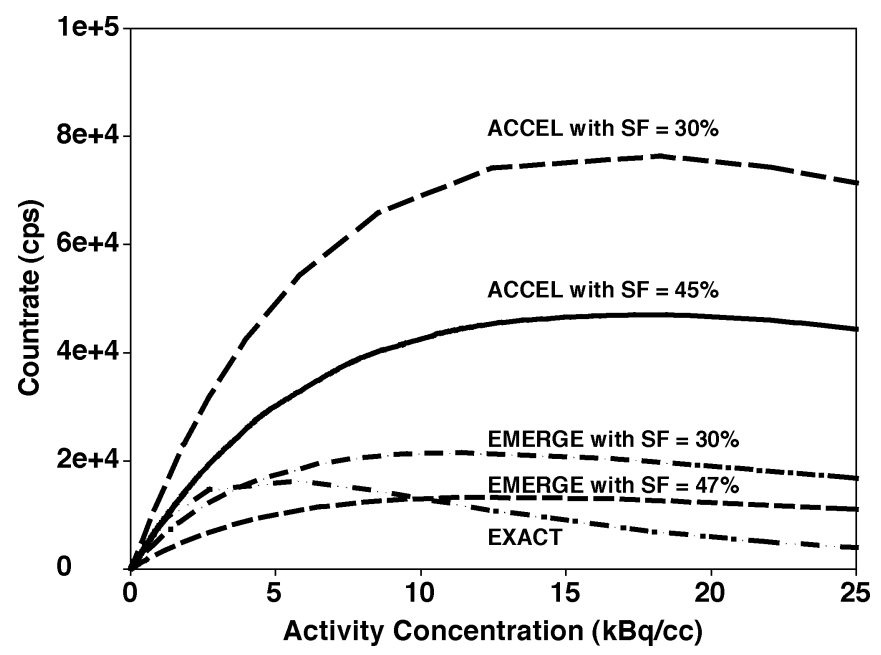

Fig. 8. Possible improvements of the LSO scanners caused by a hypothesized reduction of the scatter fraction to $30 \%$ are indicated by the comparison with the original NEC rate curves of the ACCEL, EMERGE, and EXACT.

olution parameters especially in the transverse orientation, because the DIFT results show distorted point spread images. The image space in which the point source has to be reconstructed must be zoomed so that the pixels are small enough to assure an adequate sampling. This is achieved by the 3DFBP reconstruction, but not by the DIFT method. The latter reconstructs with image pixels $2.1 \mathrm{~mm} \times 2.1 \mathrm{~mm}$ primarily and then zooms the reconstructed image to a pixel size of $0.25 \mathrm{~mm} \times 0.25 \mathrm{~mm}$. This approach is not suited to sample a point source properly and results in distorted point spread images. At PET sites where the DIFT reconstruction is the only available procedure, accurate resolution tests might be problematic. On the other hand, when we compared images of a 3-D Hoffman phantom filled with ${ }^{18} \mathrm{~F}$, which had been reconstructed with both methods, no obvious difference, which might have been related to the different reconstruction methods, could be detected.

As the resolution test is done with point sources in air, this test may have limited value for the real situation where the radioactivity is distributed in dense tissue. An additional test with the point sources positioned in water may be useful.

Most of the tests included in the NEMA NU2-2001 standard yield relative radioactivity numbers, i.e., the count rate accuracy and the image quality test. In contrast, the sensitivity test produces an absolute result. Here, the determination of the radioactivity inserted in the central tube represents a possibly critical additional variable that may introduce an error. We were not able to check the accuracy of the activimeters at all scanner sites. In the case of the $\mathrm{HR}+$, we could confirm our result in a repeated test. Regarding the EXACT and the ACCEL, our data were close to those of the manufacturer, which was obtained via a point source measurement combined with a specific calculation (personal communication).

The image quality test underlined the comparably lower plane sensitivity of high-resolution scanners like the HR+. The background in the HR+ images looks less uniform than in the other scanners [Fig. 7(c)]. Thus, the theoretically better ability of the HR+ to reveal small hot spots because of its better resolution is counteracted by its inferior background uniformity. The finding of the improved uniformity of the LSO scanner ACCEL [Fig. 7(a)] is also supported by the images of the partial ring LSO scanner EMERGE [Fig. 7(d)], which seems to be only slightly inferior to the EXACT [Fig. 7(b)], although its sensitivity is much less.

Although the images of the quality test reveal a less uniform background of the $\mathrm{HR}+$, this finding is not really mirrored in the results produced by this test (Table V). When we defined an additional large ROI with an area of $150 \mathrm{~cm}^{2}$, which was 2 $\mathrm{cm}$ axially apart from the plane with the center of the spheres, 
i.e., in a background plane, and calculated its coefficient of variance, we got $13.71 \%$ for $\mathrm{HR}+, 10.94 \%$ for the EXACT, $6.08 \%$ for the ACCEL, and $14.19 \%$ for the EMERGE. These numbers correspond quite well with the image impression. The test of the residual error in attenuation and scatter corrections with its parameter $\Delta C_{\text {lung }}$ yielded values from $28.7 \%$ to $47.8 \%$. Ideally, this value should have been zero. This result indicates the necessity of an improvement in the corrections.

This paper reports the technical characteristics of four commercial dedicated PET scanners. The data obtained can serve as a basis for rationally designing protocols of image acquisition in the clinical surrounding. The devices studied encompass a wide range of system costs; as expected, the more expensive scanners perform better than the comparatively cheaper tomographs. Cameras equipped with LSO crystals seem to be superior to those using BGO at higher tissue concentrations of radioactivity. These may especially be found in images of cerebral or cardiac FDG uptake, at the beginning of dynamic studies, or when higher tracer doses are injected. Theoretically, differences in technical performance should translate directly into differences in diagnostic accuracy. However, the proof for this assumption and an analysis of the clinical impact of this relationship can only be obtained via clinical studies.

\section{ACKNOWLEDGMENT}

The authors thank Dr. Aurich, Dr. Tosch, and Mr. Esser, BS (St. Franziskus-Hospital, Moenchengladbach, Germany) and Prof. Bossuyt and Dr. Everaert (Department of Nuclear Medicine at the Academic Hospital of the Free University of Brussels, Belgium) for their hospitality and the opportunity to perform the reported tests on the ECAT EXACT and ECAT ACCEL, respectively. Furthermore, the authors thank Mr. Pfeiffer (Siemens Medical Solutions, Erlangen, Germany) for his efficient support. The authors appreciate the help of Prof. Qaim (Institute of Nuclear Chemistry, Forschungszentrum Juelich) for critical reading of the manuscript.

\section{REFERENCES}

[1] J. S. Karp, M. E. Daube-Witherspoon, E. J. Hoffman, T. K. Lewellen, J. M. Links, W. H. Wong, R. D. Hichwa, M. E. Casey, J. G. Colsher, and R. E. Hitchens et al., "Performance standards in positron emission tomography," J. Nucl. Med., vol. 32, pp. 2342-2350, 1991.
[2] R. Guzzardi, C. R. Bellina, and B. Knoop et al., "Methodologies for performance evaluation of positron emission tomographs," J. Nucl. Biol. Meth., vol. 35, pp. 141-157, 1991.

[3] NEMA, "Performance measurements of positron emission tomographs," Nat. Elect. Manufact. Assoc., Washington, DC, NEMA Standards Pub. NU 2-1994, 1994.

[4] IEC, "Radionuclide devices-characteristics and test conditions. Part I. Positron emission tomographs," Int. Electrotech. Commiss., Geneva, Switzerland, EC Standard 61 675-1, 1998.

[5] R. A. Koeppe and G. D. Hutchins, "Instrumentation for positron emission tomography: Tomography and data processing and display systems," Sem. Nucl. Med., vol. 22, pp. 162-181, 1992.

[6] NEMA, "Performance measurements of positron emission tomographs," Nat. Elect. Manufact. Assoc., Rosslyn, VA, NEMA Standards Pub. NU 2-2001, 2001.

[7] D. L. Bailey, T. Jones, and T. J. Spinks, "A method for measuring the absolute sensitivity of positron emission tomographic scanners," Eur. J. Nucl. Med., vol. 18, pp. 374-379, 1991.

[8] K. Wienhard, L. Eriksson, S. Grootoonk, M. Casey, U. Pietrzyk, and W.-D. Heiss, "Performance evaluation of the positron scanner ECAT EXACT," J. Comput. Assist. Tomogr., vol. 16, pp. 804-813, 1992.

[9] L. Adam, H. Ostertag, H. Trojan, M. E. Bellemann, and G. Brix, "Performance evaluation of the whole-body PET scanner ECAT EXACT HR+ following the IEC standard," IEEE Trans. Nucl. Sci., vol. 44, pp. 1172-1179, June 1997.

[10] G. Brix, J. Zaers, L. Adam, M. E. Bellemann, H. Ostertag, H. Trojan, U. Haberkorn, J. Doll, F. Oberdorfer, and W. J. Lorenz, "Performance evaluation of a whole-body PET scanner using the NEMA protocol," $J$. Nucl. Med., vol. 38, pp. 1614-1623, 1997.

[11] L. Eriksson, K. Wienhard, M. Eriksson, M. E. Casey, C. Knoess, T. Bruckbauer, J. Hamill, T. Mulnix, S. Vollmar, B. Bendriem, W.-D. Heiss, and R. Nutt, "NEMA evaluation of the first and second generation of the Ecat exact and Ecat exact HR family of scanners," in Proc. IEEE Con. Medical Imaging, San Diego, CA, 2001.

[12] M. E. Daube-Witherspoon, J. S. Karp, M. E. Casey, F. D. DiFilippo, H. Hines, G. Muehllehner, V. Simcic, C. W. Stearns, L.-E. Adam, S. Kohlmeyer, and V. Sossi, "PET performance measurements using the NEMA NU 2-2001 standard," J. Nucl. Med., vol. 43, pp. 1398-1409, 2002.

[13] E. J. Hoffman, S. C. Huang, M. E. Phelps, and D. E. Kuhl, "Quantitation in positron emission computed tomography: 4. Effect of accidental coincidences," J. Comput. Assist. Tomogr., vol. 5, pp. 391-400, 1981.

[14] H. Stark, J. Woods, I. Paul, and R. Hingorani, "Direct Fourier reconstruction in computer tomography," IEEE Trans. Acoust. Speech Signal Processing, vol. ASSP-29, pp. 237-245, Feb. 1981.

[15] M. Defrise, D. W. Townsend, and R. Clack, "Three-dimensional image reconstruction from complete projections," Phys. Med. Biol., vol. 34, pp. 573-583, 1989.

[16] M. Witte, "Bewertung eines Positronen-Emissions-Tomographen mit Hilfe des aktuellen NEMA-Standards NU2-2001," Diploma-thesis, Univ. Applied Sciences Aachen, Juelich, Germany, 2002.

[17] S. C. Strother, M. E. Casey, and E. J. Hoffman, "Measuring PET scanner sensitivity: Relating count-rates to image signal-to-noise ratios using noise equivalent counts," IEEE Trans. Nucl. Sci., vol. 37, pp. 783-788, Apr. 1990.

[18] M. Lonneux, I. Borbath, A. Bol, A. Coppens, M. Sibomana, R. Bausart, M. Defrise, S. Pauwels, and C. Michel, "Attenuation correction in whole-body FDG oncological studies: The role of statistical reconstruction," Eur. J. Nucl. Med., vol. 26, pp. 591-598, 1999. 